\title{
Configuración multicriterio de células de fabricación considerando efecto sobre planes de producción
}

\author{
Multicriteria configuration of manufacturing cells considering effect on production plans
}

\author{
A. M. Castillo-Zapata , J. P. Orejuela-Cabrera , C. A. Gil-González iD
}

\begin{abstract}
Resumen-En mercados competitivos la flexibilidad es una exigencia clave para competir, la tecnología de grupos permite obtener esta flexibilidad a bajo costo, debido a que combina las ventajas de la producción en masa con las ventajas de la producción por talleres. En tal sentido este artículo tiene como objetivo proponer una metodología que integre los planes de: configuración de planta, capacidad y producción valorando múltiples criterios para seleccionar las alternativas de agrupación con mejores resultados. Para ello se proponen realizar 5 etapas, creación de familias de productos, configuración de planta, capacidad y producción, estimación de medidas de desempeño y proceso de análisis jerárquico. Como resultado, se logra una metodología consistente respecto a la sensibilidad de la evaluación AHP de las medidas de desempeño, las variaciones individuales en los criterios de las configuraciones candidatas no modifican la decisión encontrada (configuración a seleccionar). La principal conclusión es, se puede abordar el problema de la formación de células considerando un enfoque en el que se descompone el problema en sub problemas menos complejos y así incluir al tiempo múltiples criterios de agrupación orientados al entorno donde sea aplicada la metodología.
\end{abstract}

Palabras claves - Células de manufactura, Coeficientes de similaridad, Distribución de planta, Sistemas de manufactura flexible, Tecnologías de grupos.

Abstract - In competitive markets, flexibility is a key requirement to compete, group technology allows you to obtain this flexibility at a low cost, because it combines the advantages of mass production with the advantages of production by workshops This paper aims to Create a methodology that integrates the plans of: plant configuration, capacity and production, evaluating multiple criteria to select the grouping alternatives with better results. The methodology has five stages: creation of product families, configuration of plant, capacity and production, estimation of performance measures and process of hierarchical analysis. A consistent methodology is achieved regarding the sensitivity of the AHP evaluation of the performance measures, the individual variations in the criteria of the candidate configurations do not modify the decision found (configuration to be selected).

This manuscript was sent on February 12, 2019 and accepted on March 28, 2019. This work was supported by the Universidad del Valle.

A. M. Castillo-Zapata. Universidad del Valle. Ciudad Universitaria Meléndez; Calle 13 \# 100-00.

J. P. Orejuela-Cabrera. Universidad del Valle. Ciudad Universitaria Meléndez; Calle 13 \# 100-00. juan.orejuela@ correounivalle.edu.co.
The main conclusion, it is possible to address the problem of cell formation considering an approach in which the problem is broken down into less complex sub-problems and thus include multiple grouping criteria at the time oriented to the environment where the methodology is applied

Index Terms-Group technologies, Flexible manufacturing systems, Manufacturing cells, Similarity coefficients, Facility layout.

\section{INTRODUCCIÓN}

E n mercados competitivos, la flexibilidad es una exigencia clave para competir y para ello se deben mantener, sistemáticamente ventajas comparativas que le permitan alcanzar, sostener y mejorar una determinada posición en el mercado, optando así por estrategias para integrar sus capacidades y recursos con la aplicación de nuevas tecnologías. Dentro de estas tecnologías existe la Tecnología de grupos, la cual se basa fundamentalmente en que las empresas generen grupos de trabajos (células de manufactura) estables con operarios y/o maquinaria que tienen la misión de hacer un producto terminado de la primera a la última operación buscando la flexibilidad a bajo costo, combinando las ventajas de la producción en masa con las ventajas de la producción por talleres.

La Tecnología de Grupos (TG) convierte sistemas de producción convencionales en sistemas flexibles de manufactura, puesto que es una filosofía de fabricación en la que productos similares se identifican y agrupan conjuntamente con el fin de aprovechar sus similitudes en el proceso de diseño y fabricación. La complejidad para realizar la formación de las células de manufactura, en parte se sustenta en la existencia de diversas configuraciones de agrupación, entre las cuales se debe buscar una alternativa con mejores resultados para el entorno productivo.

C. A. Gil-González, Universidad de Chile. Av. Libertador Bernardo O'Higgins 1058, Santiago de Chile. cristiam.gil@ing.uchile.cl. 
Todas estas configuraciones de agrupación tienen características (ventajas y desventajas) que no necesariamente pueden ser tratadas en una función objetivo a optimizar. Existen múltiples criterios adicionales en los sistemas de manufactura flexible que eventualmente toman relevancia de acuerdo al entorno de aplicación o al contexto de la instancia. Generalmente en la literatura estos criterios son abordados individualmente, no como un conjunto que afecta simultáneamente el sistema.

Por ejemplo, en [1] es medido el impacto del criterio de backorder. Por lo anterior, la metodología que se presenta en este estudio propone medidas adicionales de desempeño para valorar estas alternativas de agrupación, compararlas y seleccionar la más apropiada según indicadores relacionados con el desempeño de del taller o planta, dado un entorno de manufactura específico.

En la literatura se pueden identificar diferentes trabajos que buscan integrar el problema de formación de células y su relación con el desempeño del taller, algunas de las relaciones que se presentan buscan involucrar la formación de células con otras decisiones tales como la planeación de la producción, la distribución de la planta y la definición de capacidad. Estos trabajos evidencian la importancia de decisión de agrupación de productos o máquinas con otros problemas de toma decisiones con el fin de mejorar los desempeños en el taller.

La integración entre la formación de células y los planes de producción es una de las más trabajados, en [2] por ejemplo, se realiza un modelo de programación lineal entera que considera la formación de células en el marco de un problema de planificación de la producción en un sistema hibrido, sin embrago a pesar de que el trabajo involucra la sostenibilidad como principal factor el modelo de toma de cesiones es mono objetivo.

En [3] se reconoce que la integración del problema de formación de células y la planeación de la producción es un problema $\mathrm{Np}$-hard y para resolverlo plantean un algoritmo meta heurístico basado en algoritmos genéticos. El problema minimiza los costes de compra de la máquina, el costo de manejo de materiales, el costo de configuración y reconfiguración de células que se recogen en una sola función de costo.

Otro algoritmo genético es empleado en [4] para resolver un modelo matemático que minimiza el costo total, considerando diferentes aspectos del sistema de manufactura, tales como la configuración de células, el problema se la asignación de las máquinas, el problema del enrutamiento de las partes y el problema de la planeación de la producción.

En [5] se plantea la importancia de integrar el diseño de sistemas de fabricación celular con las decisiones tácticas relacionadas con aspectos de la planeación de la producción.
Para ello plantean un modelo matemático que busca seleccionar y optimizar la asignación de partes y máquinas a las diferentes células y también busca definir la cantidad de partes a producir en cada periodo. A pesar de que este trabajo considera cinco funciones objetivo todas son consolidadas en una sola función global de costo.

Reconociendo la importancia y la complejidad que implica la integración de la planeación de la producción y en diseño de los procesos en sistemas de manufactura celular en [6] se propone una metodología que resume los pasos necesarios para planificar un producto desde el diseño del proceso para la orden de fabricación hasta la entrega al cliente.

La integración de la formación de las células con la planificación se da en diferentes niveles de planeación tal como en [7] en el que se busca el cumplimiento eficiente de un programa Maestro de producción, o como en [8] y [9] que se relacionan con la programación fina de las operaciones $\mathrm{o}$ scheduling.

La integración se da con diferentes estrategias o enfoques de planeación tal como en [10] donde se realiza la formación de células en el contexto de la planeación ajustada o Lean Manufacturing o en [11] que se plantea desde un enfoque sistemático o en [12] que se plantea desde un enfoque práctico. Por otro lado, la integración de la formación de células con distribución de planta y la planificación de la producción se puede ver en [13].

El problema de formación de células o de agrupación de máquinas, en sí mismo tiene sus propias necedades y en ese sentido se han planteado diversas investigaciones alrededor de aspectos muy específicos, por ejemplo, en [14] donde se analizan los valores para los umbrales de similitud y se hace una propuesta para su determinación.

En [15] se desarrolla una metodología multi atributo en la que se utiliza un algoritmo de agrupación y el AHP para escogerla mejor alternativa de cara a los objetivos propuestos por los expertos. Sin embargo, los impactos de las afectaciones de las agrupaciones en el plan de producción o en las medidas del taller se deja para la valoración subjetiva. En [16] se trabaja el problema desde un enfoque multi-objetivo. El problema tratado, considera la demanda probabilística y la confiabilidad de las máquinas. Busca la minimización de tres objetivos: el costo total, la utilización y la máxima tasa de fallo del sistema.

Un número importante de trabajos se has desarrollado alrededor la importancia de involucrar el enfoque de múltiples objetivos o múltiples criterios en el problema de agrupación. Diferentes herramientas han sido empleadas para abordar este enfoque: multi atributo con TOPSIS [17], multi atributo con AHP [18] y [15], multi objetivo con NSGAII y épsilon restricciones [16], TOPSIS y PLE [17]

A partir de la revisión planteada se identifica que el problema 
de agrupación de células es vigente y tiene nuevos desafíos que le impregnan las necesidades cambiantes del mercado, obligándolo a ser visto de una manera sistémica. Que lo ubica como un sub problema que se relaciona y se integra con otros sub problemas, obligándolo a ser evaluado desde diferentes dimensiones inherentes a estos. En ese contexto se identifican diferentes trabajos que involucran ya sea la integración o la evaluación múltiple de la agrupación desde diferentes enfoques y reconocen la complejidad de estos. En ese sentido el presente trabajo aborda el problema de la formación de células considerando un enfoque en el que se descompone el problema en sub problemas menos complejos. Y así poder incluir al tiempo múltiples criterios de agrupación orientados al taller, la integración con el plan de producción y plan de capacidad a mediano plazo.

\section{MATERIALES Y MÉTODOS}

Este trabajo integra la configuración de planta, capacidad y producción en el mediano plazo en ambientes de manufactura flexible en tres etapas, ver fig. 1.

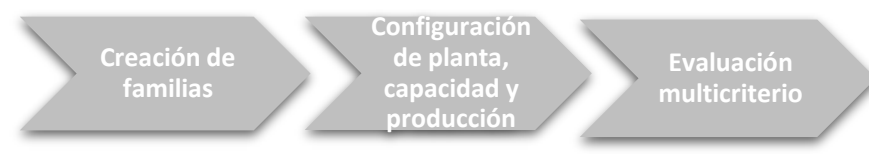

Fig. 1. Propuesta metodológica para solución del problema bajo estudio. Fuente: Elaboración propia

La información obtenida en cada etapa es input para la etapa siguiente. A continuación, son descritas las etapas de la metodología.

\section{A. Creación de familias}

En esta etapa de la metodología se hace uso de la herramienta conceptual de tecnología de grupos, es resuelto el problema de la agrupación de partes en familias a través de un modelo pmediana. Este modelo emplea como coeficientes de agrupación las medidas de distancia y similaridad basadas en las matrices de incidencia máquina vs producto, en lo que sigue es explicado su cálculo y posterior uso en el modelo p-mediana.

\section{1) Cálculo de coeficientes de agrupación}

Una matriz de incidencia máquina vs producto, nos indica los centros de trabajo en donde se elaboran los productos y el orden en que se deben realizar las operaciones para la obtención de cada uno de éstos, en donde el valor de 1 significa la realización del producto en el centro de trabajo y el valor de 0 la no realización.

Los coeficientes de similaridad son una medida de correspondencia o semejanza entre los productos que van a ser agrupados. La estrategia más común consiste en medir la equivalencia en términos de la distancia entre los pares de productos. Los productos con distancias reducidas entre ellos son más parecidos entre sí que aquellos que tienen distancias mayores y se agruparán, por tanto, dentro de la misma célula. De esta manera, cualquier producto puede compararse con cualquier otro producto a través de la medida de similaridad o de distancia. Para su cálculo se emplean las matrices de incidencia máquina vs producto.

Con la matriz de incidencia máquina vs producto, se computan las medidas de distancias y similaridad entre los productos. En los modelos más comunes los coeficientes de similaridad se calculan sobre caracteres de 2 estados, cada uno de los cuales se registra como 1 o 0 (presencia o ausencia). De forma que se puede llegar a obtener una tabla de contingencia como se muestra en la fig. 2.

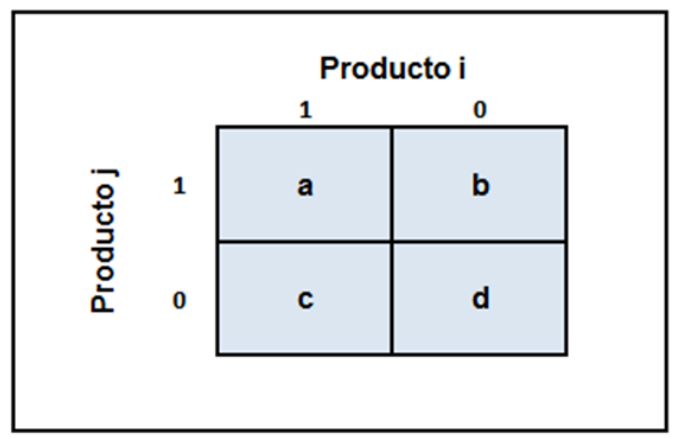

Fig. 2. Tabla de contingencia. Fuente: [19]

Dónde: a es el número de máquinas presentes en ambos productos, b es el número de máquinas presentes en el producto “i” pero no “j”, c es el número de máquinas presentes en el producto “j” pero no “i”” y d es el número de máquinas ausentes en ambos productos.

Posterior a la tabla de contingencia se construyen los coeficientes de similaridad (los más comunes son presentados en la tabla 1), los cuales indican la relación que existe entre dos productos. Dependiendo del coeficiente seleccionado se podrían obtener resultados diversos. Una excelente revisión sobre los coeficientes de similaridad puede ser encontrada en [20].

TABLA I.

DEFINICIÓn De COEFICIENTES DE Similaridad. Fuente: ElaboraCión PROPIA.

\begin{tabular}{llc}
\hline \hline \multicolumn{1}{c}{ Autor } & \multicolumn{1}{c}{ Definición } & \multicolumn{1}{c}{ Rango } \\
Jaccard & $\mathrm{a} /(\mathrm{a}+\mathrm{b}+\mathrm{c})$ & {$[0,1]$} \\
Hamann & {$[(\mathrm{a}+\mathrm{d})-(\mathrm{b}+\mathrm{c})] /(\mathrm{a}+\mathrm{b}+\mathrm{c}+\mathrm{d})$} & {$[-1,1]$} \\
Yule & $(\mathrm{ad}-\mathrm{bc}) /(\mathrm{ad}+\mathrm{bc})$ & {$[-1,1]$} \\
Simple Matching & $(\mathrm{a}+\mathrm{d}) /(\mathrm{a}+\mathrm{b}+\mathrm{c}+\mathrm{d})$ & {$[0,1]$} \\
Sorenson & $2 \mathrm{a} /(2 \mathrm{a}+\mathrm{b}+\mathrm{c})$ & {$[0,1]$} \\
Rogers and & $(\mathrm{a}+\mathrm{d}) /(2(\mathrm{~b}+\mathrm{c})+\mathrm{a}+\mathrm{d})$ & {$[0,1]$} \\
Tanimoto & & \\
\hline \hline
\end{tabular}




\begin{tabular}{lll}
\hline \hline Sokal and Sneath & $2(\mathrm{a}+\mathrm{d}) /(2 \mathrm{a}+\mathrm{d})+\mathrm{b}+\mathrm{c})$ & {$[0,1]$} \\
Rusell and Rao & $\mathrm{a} /(\mathrm{a}+\mathrm{b}+\mathrm{c}+\mathrm{d})$ & {$[0,1]$} \\
$\begin{array}{l}\text { Baroni-Urbani } \\
\text { and Buser }\end{array}$ & {$\left[\mathrm{a}+(\mathrm{ad})^{1 / 2}\right] /\left[\mathrm{a}+\mathrm{b}+\mathrm{c}+(\mathrm{ad})^{1 / 2}\right]$} & {$[0,1]$} \\
Phi & $(\mathrm{ad}-\mathrm{bc}) /[(\mathrm{a}+\mathrm{b})(\mathrm{a}+\mathrm{c})(\mathrm{b}+\mathrm{d})(\mathrm{c}+\mathrm{d})]^{1 / 2}$ & {$[-1,1]$} \\
$\begin{array}{l}\text { Ochiai } \\
\text { Distancia de }\end{array}$ & $\mathrm{a} /[(\mathrm{a}+\mathrm{b})(\mathrm{a}+\mathrm{c})]^{1 / 2}$ & {$[0,1]$} \\
Hamming & $(\mathrm{b}+\mathrm{c})$ & {$[0, \infty)$} \\
& & \\
Distancia de & $(\mathrm{a}+\mathrm{c})^{1 / 2}$ & {$[0, \infty)$} \\
Minkowski & & \\
& & \\
\hline \hline
\end{tabular}

\section{2) Modelo 1: P-mediana}

Entre los modelos de programación matemática para la resolución de problemas tecnología de grupos se destacan los modelos tipo p-mediana. Este tipo de modelos se emplea para agrupar $\mathrm{n}$ partes en $\mathrm{p}$ familias de partes, pero requiere de la especificación de un número límite de familias de partes a ser creadas.

La metodología de este tipo de modelo, permite desarrollar una serie de consideraciones de diferentes factores productivos del mundo real tales como: secuencia de operación, producción de cantidades (volúmenes) y/o grupos de partes. El modelo PMediana abordado en este documento [21] resuelve el problema de la agrupación de productos en familias basándose en las medidas de distancia y similaridad presentados en el literal anterior.

El resultado esperado de este modelo es un conjunto de variables binarias que indican si un producto debe pertenecer o no a una familia, lo cual define que productos se deben agrupar en $p$ familias definidas previamente. Estos dos outputs posteriormente parametrizaran el modelo de configuración de planta, capacidad y producción.

\section{B. Configuración de planta, capacidad y producción: modelo propuesto}

El modelo de programación lineal entera mixta de [1] es tomado como base para el desarrollo de un modelo de programación no lineal, el cual se encargará de la integración de la configuración de planta con tecnología de grupos, de la planeación de la capacidad y de la planeación de la producción.

A continuación, se describe parte la notación del modelo: conjuntos, variables y parámetros que son empleados para la definición en especial de las medidas de desempeño que se propondrán y de restricciones específicas que se explicarán más adelante.

\section{1) Índices}

- $\quad \mathrm{k}$ es el índice para las operaciones.

- $\quad$ es el índice para los productos.

- $\quad$ f es el índice para las familias.

- m es el índice para los tipos de máquinas.
- $\quad$ g es el índice para los grupos.

- $\mathrm{t}$ es el índice para los períodos de tiempo en el horizonte de planeación.

\section{2) Parámetros}

- $\quad \mathrm{TO}_{\mathrm{km}}$ es el tiempo de producción de la operación k en la máquina tipo $\mathrm{m}$.

- $\mathrm{CAM}_{\mathrm{m}}$ es la capacidad valorada en tiempo de la máquina tipo $\mathrm{m}$.

- Horizonte es el número total de períodos de tiempo de la planeación.

- $\mathrm{DEM}_{\mathrm{pt}}$ es la demanda del producto p en el período de tiempo t.

- TotalOp es el número total de operaciones en la planta.

\section{3) Variables}

- $\quad \mathrm{P}_{\mathrm{pt}}$ es la cantidad a fabricar del producto $\mathrm{p}$ en el periodo $t$.

- $\quad \mathrm{NM}_{\mathrm{mg}}$ es el número de máquinas tipo m asignadas al grupo $\mathrm{g}$.

- $\mathrm{B}_{\mathrm{pt}}$ es la cantidad de pedidos pendientes del producto $\mathrm{p}$ en el periodo $\mathrm{t}$.

- $\quad \mathrm{V}_{\mathrm{kg}}^{+}$es una variable binaria donde es igual a 1 si operaciones sucesivas de un producto $\mathrm{k}$ se asignan a grupos diferentes. Es igual a 0 de lo contrario.

- $Z_{g}$ es una variable binaria donde es igual a 1 si se usa el grupo g. Es igual a 0 de lo contrario.

- $X_{\mathrm{kg}}$ es una variable binaria donde es igual a 1 si se asigna la operación $\mathrm{k}$ al grupo g. Es igual a 0 de lo contrario.

4) Cambios del modelo lineal al no lineal. El modelo propuesto en este estudio se diferencia con el modelo planteado por [1] en el siguiente conjunto de restricciones:

$$
\begin{gathered}
\sum_{\text {keOperación }} \mathrm{Q}_{\mathrm{kgt}} * \mathrm{TO}_{\mathrm{km}} \leq \mathrm{CAM} * \mathrm{NM}_{\mathrm{mg}} \forall \mathrm{m}, \mathrm{g}, \mathrm{t} \\
\mathrm{Q}_{\mathrm{kgt}} \leq \mathrm{X}_{\mathrm{kg}} * \sum_{\mathrm{t} \in \mathrm{Tiempo}} \operatorname{DEM}_{\mathrm{pt}} \forall \mathrm{p}, \mathrm{k} \in \operatorname{RutaP}(\mathrm{p}), \mathrm{g}, \mathrm{t} \\
\mathrm{Q}_{\mathrm{kgt}} \leq \mathrm{P}_{\mathrm{pt}} \forall \mathrm{p}, \mathrm{k} \in \operatorname{RutaP}(\mathrm{p}), \mathrm{g}, \mathrm{t} \\
\mathrm{P}_{\mathrm{pt}}+\mathrm{Q}_{\mathrm{kgt}} \leq\left(1-\mathrm{X}_{\mathrm{kg}}\right) * \sum_{\mathrm{t} \in \text { Tiempo }} \operatorname{DEM}_{\mathrm{pt}} \forall \mathrm{p}, \mathrm{k} \\
\in \operatorname{RutaP}(\mathrm{p}), \mathrm{g}, \mathrm{t}
\end{gathered}
$$

Las cuales aseguran el cumplimiento de los requerimientos de capacidad de las máquinas y define las cantidades a producir de las operaciones para el cumplimiento de la demanda durante el horizonte de planeación. Gracias a la flexibilidad en la modelación que permite la programación no lineal este conjunto es reemplazado por la expresión 5 y fue abordado por software de optimización no lineal Lingo. 


$$
\sum_{\mathrm{p}}^{\mathrm{P}} \sum_{k=1}^{K} P_{p t} * \mathrm{TO}_{\mathrm{km}} * X_{k g} \leq \mathrm{CAM}_{\mathrm{m}} * \mathrm{NM}_{\mathrm{mg}} \forall m, g, t
$$

\section{Evaluación multi criterio}

En esta etapa de la metodología se establecen una serie de criterios los cuales ayudan a contrastar las cargas entre centros de trabajo y actividades a realizar en cada uno de ellos, estos nos permitirán evaluar el desempeño y la eficiencia de las configuraciones posibles encontradas por el modelo propuesto. Subsiguientemente, para validar una de estas agrupaciones como la más apropiada al entorno productivo que se estudie se hace necesario el desarrollo de la metodología AHP.

\section{1) Estimación de medidas de desempeño}

Debido a que existe un gran número importante de coeficientes de similaridad y existen diferentes números de familias a definir en el modelo de p-mediana, es posible encontrar múltiples agrupaciones. Por ello es importante establecer una estrategia que permita seleccionar cual es la mejor a la luz de diferentes criterios los cuales están relacionados directamente con el desempeño del taller. Por esto se proponen medidas adicionales de desempeño para comparar estas alternativas de configuración entre si incorporando múltiples métricas, las medidas que se proponen son las siguientes:

1.1) Utilización Global (U). Es el porcentaje global de utilización de tiempo de máquina. Las máquinas son versátiles y pueden manufacturar una gran variedad de partes, cada orden de producción se compone de un gran número de productos de un volumen de producción bajo a medio. Los tiempos de espera se mantienen al mínimo, por lo tanto, se espera que la utilización de un sistema flexible de manufactura se incremente significativamente. Entre más alto el indicador mejor porcentaje de utilización tiene el sistema.

$$
U=\frac{\sum_{p} \sum_{k(p)} \sum_{m}\left(T O_{k m} * \sum_{t} P_{p t}\right)}{\sum_{g} \sum_{m}\left(N M_{m g} * C A M_{m}\right) * \text { Horizonte }}
$$

1.2) Nivel de servicio (S). Es el porcentaje de las unidades satisfechas a tiempo y lleva a proporcionar un servicio satisfactorio al cliente final, quien busca puntualidad en la entrega de los productos además de una excelente calidad de los mismos.

$$
S=1-\frac{\sum_{p} \sum_{t} B_{p t}}{\sum_{p} \sum_{t} D E M_{p t}}
$$

1.3) Movimiento entre células (M). Es el número medio de movimientos hechos fuera de la célula, dado la asignación de máquinas y operaciones en células, por cada unidad de producto demandada. se considera el movimiento intercelular al salir del grupo inicialmente asignados con el objetivo de minimizar el costo de manipulación de productos inter-células.

$$
M=\frac{\sum_{t} \sum_{p} \sum_{g} P_{p t} * \sum_{k(p)} V_{k g}^{+}}{\sum_{t} \sum_{p} D E M_{p t}}
$$

1.4) Tiempo en inventario (T). Porcentaje global de tiempo productivo que permanecen lo productos como inventario en proceso, esto puede afectar el desempeño del sistema de manufactura debido a requerimientos de espacio adicionales y problemas de flujo de materiales.

$$
\begin{aligned}
& T=\frac{\sum_{p} \sum_{t} P_{p t} * \sum_{m} \sum_{k(p)} T I_{k m}}{\sum_{g} \sum_{m}\left[N M_{m g} * C A M_{m}\right]} \quad \forall T I_{k m}>0 \\
& \text { donde } T I_{k m}=\left(T O_{k+1, m}-T O_{k, m}\right)
\end{aligned}
$$

1.5) Inventario máximo en proceso (IM). Es la necesidad máxima de requerimiento de almacenamiento valorado en unidades de producto en proceso que eventualmente el sistema de manufactura podría acumular dado los planes de configuración de planta, capacidad y producción.

$$
\begin{array}{r}
I M=\max _{t \in T}\left[\sum_{p} \max _{k \in R u t a(p)}\left(\frac{T I_{k m}}{T O_{k m}}\right)\right. \\
\left.* D E M_{p t}\right] \forall T I_{k m}>0
\end{array}
$$

1.6) Desviación de las operaciones (DK). Es la máxima relación entre el tiempo empleado por las operaciones asociadas a cada grupo de la configuración evaluada y el número de operaciones totales. Este indicador permite tener una concepción simplificada de la concentración de trabajo en la configuración como una medida de riesgo.

$D K=\max _{g \in G}\left(\sum_{k \in g} T O_{k m} /\right.$ TotalOP $)$

1.7) Capacidad de las máquinas en los grupos (CM). En términos de la industria manufacturera en general, la capacidad se refiere al volumen de producción que se puede obtener con los recursos disponibles de una empresa en determinado momento (recursos como dinero, equipos, personal, instalaciones, etc.). En este caso el recurso son las máquinas, la manera de obtenerla es calcular cuántas unidades de producto puede la empresa fabricar en un tiempo y multiplicar eso por la capacidad disponible.

$$
C M=\sum_{g} \sum_{m}\left(N M_{m g} * C A M_{m}\right)
$$


El objetivo es seleccionar la mejor configuración de una célula de manufactura de todas las opciones disponibles. No obstante, dada la variedad de alternativas de configuración final posibles y la no comparabilidad de las unidades de medida de los criterios de desempeño propuesta, para llegar a un consenso respecto a la mejor alternativa disponible se aplicará la técnica del Proceso Analítico Jerárquico (AHP, por sus siglas en ingles), esta metodología de comparación se encuentra descrita detalladamente en [22].

\section{CASO ESTUDIO}

A partir de los datos presentados por [1] se diseña la siguiente instancia en la cual se omiten los detalles del proceso de producción de los productos por tratarse de un caso de evaluación y para simplificar la complejidad computacional asociada a la solución del problema. La metodología en este documento nace como una iniciativa enfocada en empresas del sector de las artes gráficas.

\section{A. Características relacionadas con los productos}

Las industrias pertenecientes al sector artes gráficas, en lo que respecta al área publi-comercial, se caracterizan por ser empresas que trabajan con un alto nivel de personalización en lo referente a los acabados de cada uno de sus productos. Por esta razón las máquinas utilizadas en este sector industrial son de tipo universales, permitiendo elaborar pedidos con altos niveles de personalización, lo que implica gran diversidad de productos.

No obstante, para factibilidad en la implementación de esta metodología, se tomaron seis tipos productos con mayor importancia para una empresa del sector, estos productos son: revistas, volantes, plegables, carpetas, libros y cuadernos. En la tabla 2 se puede apreciar las unidades demandas de los 6 productos durante 6 periodos de horizonte de planeación y en la tabla 3 se resume el número de unidades en inventario y pendientes por entregar al inicio del horizonte de planeación.

TABLA II.

UNIDADES DEMANDADAS POR PRODUCTO Y PERIOD. FUENTE: ELABORACIÓN PROPIA.

\begin{tabular}{cccccccc}
\multicolumn{10}{c}{ PROPIA. } \\
\hline \hline & & $\mathbf{1}$ & $\mathbf{2}$ & $\mathbf{3}$ & $\mathbf{4}$ & $\mathbf{5}$ & $\mathbf{6}$ \\
& Revistas & 130 & 45 & 100 & 130 & 45 & 100 \\
& Volantes & 150 & 50 & 100 & 150 & 50 & 100 \\
& Plegables & 210 & 50 & 200 & 210 & 50 & 200 \\
& Carpetas & 150 & 65 & 120 & 150 & 65 & 120 \\
& Libros & 170 & 70 & 100 & 120 & 40 & 150 \\
\hline \hline
\end{tabular}

\begin{tabular}{lllllll} 
Cuadernos & 200 & 50 & 150 & 110 & 50 & 100 \\
\hline
\end{tabular}

TABLA III.

UNIDADES DE INVENTARIO Y BACKORDER INICIALES. FUENTE: ELABORACIÓN PROPIA.

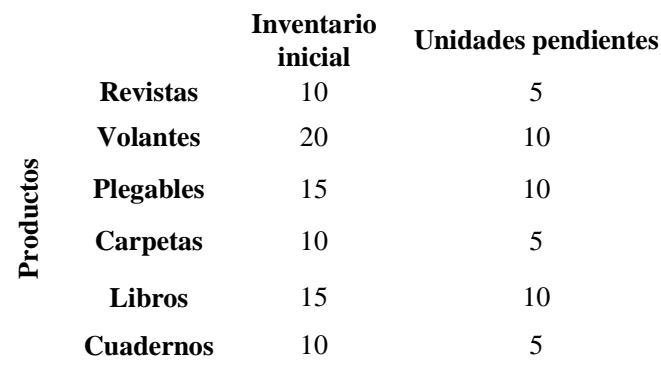

\section{B. Características relacionadas con el proceso de producción}

En la fig. 3 se muestra el proceso productivo para la elaboración de los seis productos mencionados los cuales requieren de una gran variedad de máquinas y estaciones de trabajo para su fabricación, lo cual se resume en 14 operaciones realizadas en 4 máquinas tipo diferentes. Las estaciones de trabajo son presentadas a continuación: 


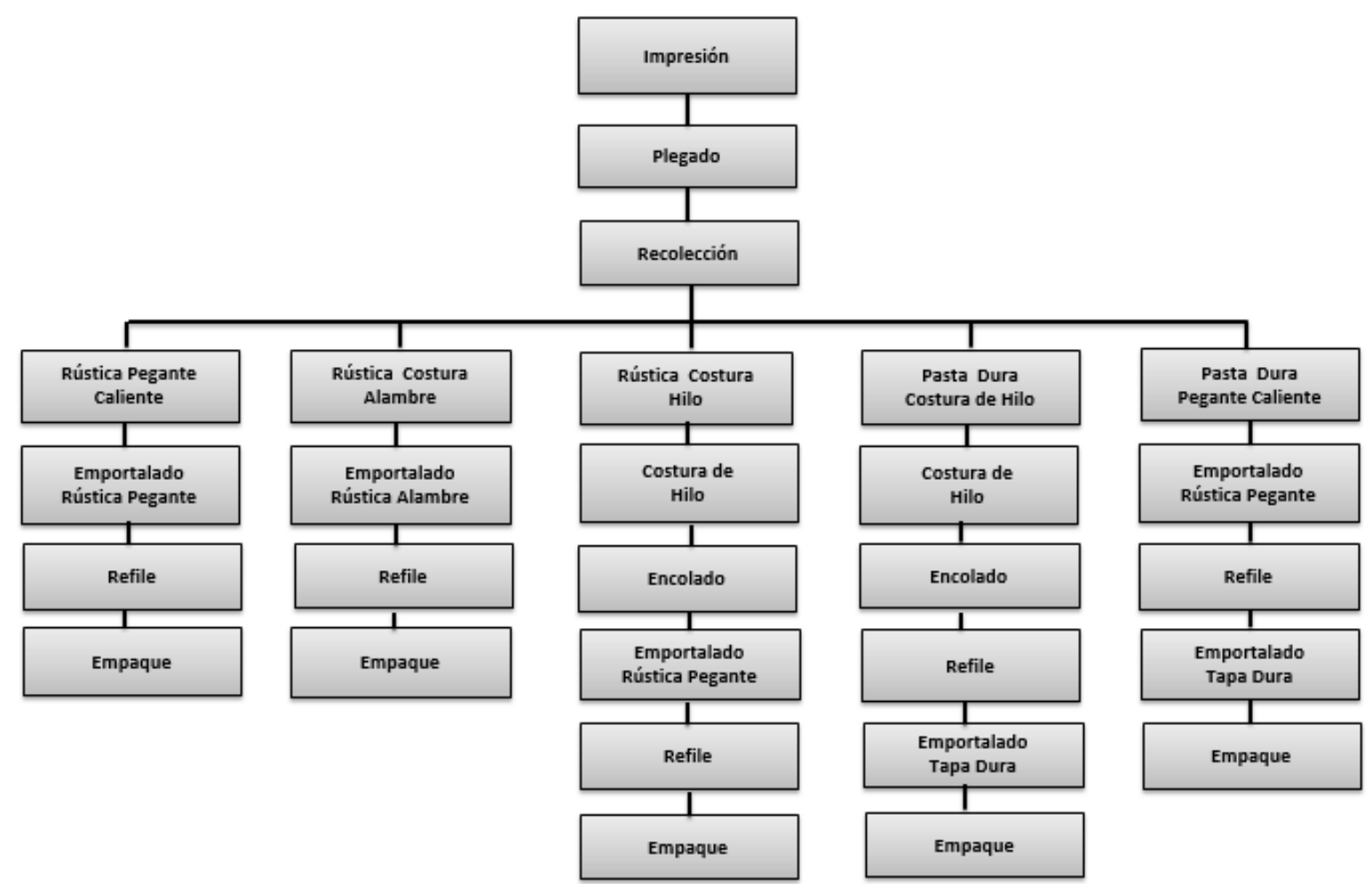

Fig. 3. Diagrama del proceso productivo. Fuente: Elaboración propia.

En la tabla 4 se presentan las máquinas o centros de trabajo (CT) en donde se elaboran los seis productos y el orden en que se deben realizar las operaciones para la obtención de cada uno de éstos, en donde el valor de 1 significa la realización del producto en el centro de trabajo y el valor de 0 la no realización del producto en el centro de trabajo. Esta información servirá como insumo para la fabricación de los coeficientes de similaridad mas adelante.

TABLA IV

MATRIZ INCIDENCIA MÁQUINA- PRODUCT

\begin{tabular}{|c|c|c|c|c|c|}
\hline & & \multicolumn{4}{|c|}{ Máquinas } \\
\hline \multirow{7}{*}{ 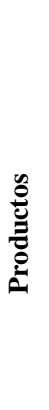 } & & 1 & 2 & 3 & 4 \\
\hline & Revistas & 1 & 0 & 0 & 1 \\
\hline & Volantes & 0 & 1 & 0 & 0 \\
\hline & Plegables & 0 & 1 & 1 & 1 \\
\hline & Carpetas & 0 & 0 & 1 & 0 \\
\hline & Libros & 0 & 1 & 0 & 0 \\
\hline & Cuadernos & 1 & 0 & 1 & 0 \\
\hline
\end{tabular}

\section{Resultados}

\section{A. Cálculo de coeficientes de Similiaridad}

Para el caso estudio, fueron calculados todas las matrices de asociación de acuerdo a los coeficientes de similaridad presentados en la tabla 1 . A continuación, en la tabla 5 y 6 , son presentados, a modo de muestra, los resultados de en bases a los coeficientes de los autores Jaccard y de Hamann.

TABLA V

MATRIZ DE ASOCIACIÓN DE JACCARD.

\begin{tabular}{ccccccc}
\hline \hline Productos & $\mathbf{1}$ & $\mathbf{2}$ & $\mathbf{3}$ & $\mathbf{4}$ & $\mathbf{5}$ & $\mathbf{6}$ \\
$\mathbf{1}$ & 1 & & & & & \\
$\mathbf{2}$ & 0 & 1 & & & & \\
$\mathbf{3}$ & 0,25 & 0,33 & 1 & & & \\
$\mathbf{4}$ & 0 & 0 & 0,33 & 1 & & \\
$\mathbf{5}$ & 0 & 1 & 0,33 & 0 & 1 & \\
$\mathbf{6}$ & 0,33 & 0 & 0,25 & 0,50 & 0 & 1 \\
\hline \hline
\end{tabular}


TABLA VI

MATRIZ ASOCIACIÓN DE HAMANN

\begin{tabular}{ccccccc}
$\mathbf{1}$ & 1 & & & & & \\
$\mathbf{2}$ & $-0,5$ & 1 & & & & \\
$\mathbf{3}$ & $-0,5$ & 0 & 1 & & & \\
$\mathbf{4}$ & $-0,5$ & 0 & 0 & 1 & & \\
$\mathbf{5}$ & $-0,5$ & 1 & 0 & 0 & 1 & \\
$\mathbf{6}$ & 0 & $-0,5$ & $-0,5$ & 0,5 & $-0,5$ & 1 \\
\hline \hline
\end{tabular}

\section{B. Modelo P-mediana}

El modelo p-mediana generó las siguientes configuraciones de familias a cada uno de los coeficientes de similaridad propuestos para los diferentes valores de $\mathrm{P}$.

La tabla 7, presenta, los coeficientes propuestos por los autores: Jaccard, Ochiai y Baroni-Urbani and Buser coinciden en la configuración 1 para cuando $P$ toma el valor de 3. Esta configuración consta de 3 familias formadas de la siguiente forma: $\mathrm{F} 1=(\mathrm{P} 1 \mathrm{P} 4 \mathrm{P} 5) \mathrm{F} 2=(\mathrm{P} 2 \mathrm{P} 6)$ y F3= (P3).

TABLA VII

NÚMERO DE CONFIGURACIONES DE FAMILIAS RESULTANTES PARA CADA TIPO DE COEFICIENTE DE SIMILARIDAD DE ACUERDO AL VALOR DE P

\begin{tabular}{|c|c|c|c|c|c|c|}
\hline Coeficientes & $\mathbf{P}=\mathbf{1}$ & $\mathbf{P}=\mathbf{2}$ & $\mathbf{P}=\mathbf{3}$ & $P=4$ & $P=5$ & $P=6$ \\
\hline Jaccard & & & & & & \\
\hline Ochiai & & & 1 & & 1 & \\
\hline \multicolumn{7}{|l|}{ Baroni-Urbani and Buser } \\
\hline \multicolumn{7}{|l|}{ Hamann } \\
\hline Yule & & 1 & & 1 & & \\
\hline Simple Matching & & & 2 & & & \\
\hline Rogers and Tanimoto & 1 & & & & 2 & 1 \\
\hline \multicolumn{7}{|l|}{ Phi } \\
\hline Sorenson & & 2 & 3 & 2 & & \\
\hline Sokal and Sneath & & 3 & 4 & 3 & & \\
\hline $\begin{array}{l}\text { Distancia de Hamming } \\
\text { Distancia de Minkowski }\end{array}$ & & 4 & 5 & 4 & 3 & \\
\hline
\end{tabular}

\section{Configuración de planta, capacidad y producción}

De este modelo se logran obtener las operaciones que son asignadas a cada familia de productos, el tipo de máquina y la cantidad requerida que ejecutaran estas operaciones en cada configuración de cada valor de P. A continuación, de forma ilustrativa, se muestran las configuraciones finales de planta cuando $\mathrm{P}$ toma el valor de 5 , las figura 4,5 y 6 presentan respectivamente las configuraciones uno, dos y tres. FAMILIA 1

GRUPOS

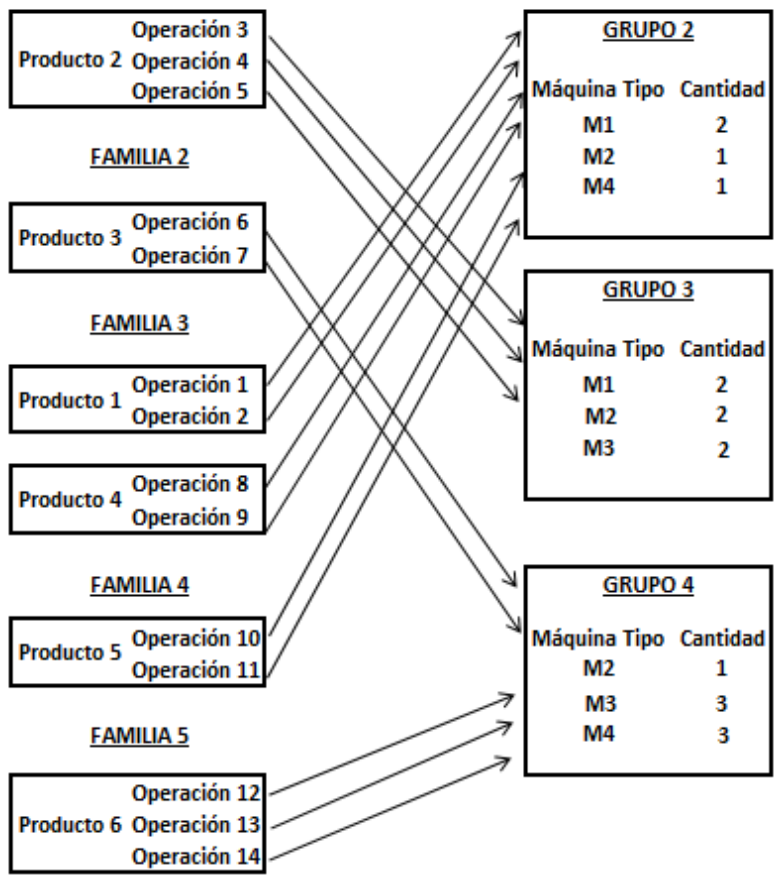

Fig. 4. Configuración 1 para $\mathrm{P}=5$. Fuente: Elaboración propia.

FAMILIA 1

GRUPOS

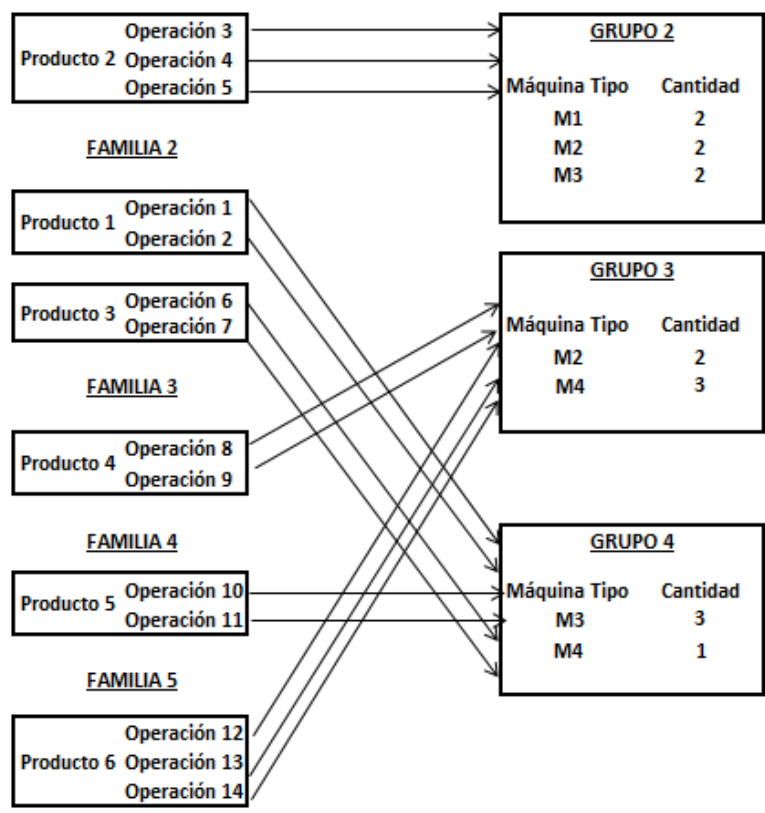

Fig. 5. Configuración 2 para $\mathrm{P}=5$. Fuente: Elaboración propia. 


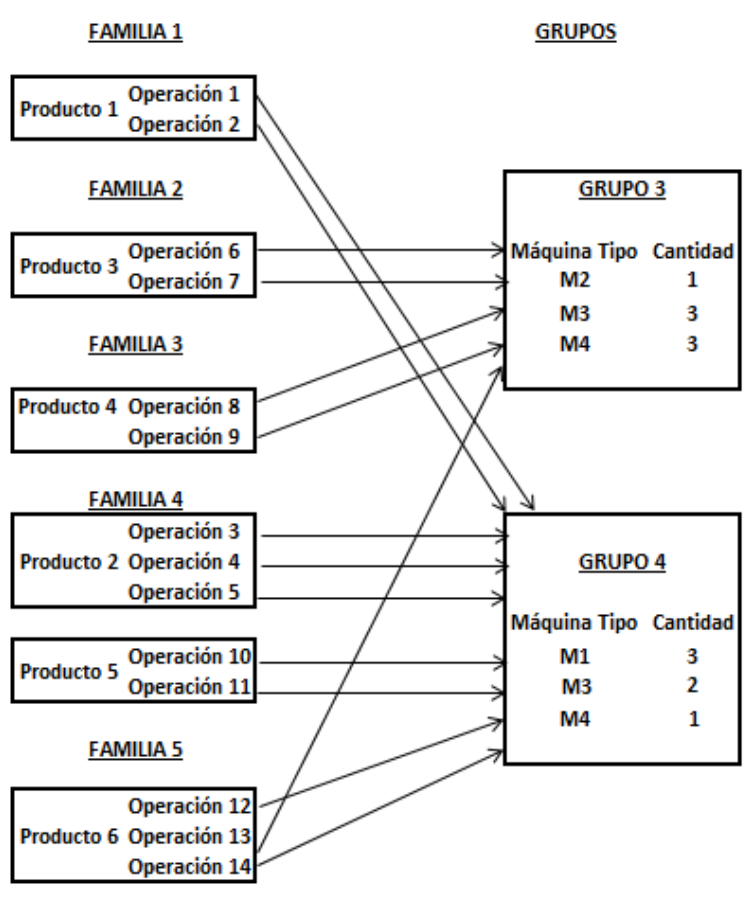

Fig. 6. Configuración 3 para $\mathrm{P}=5$. Fuente: Elaboración propia.

\section{Estimación de medidas de desempeño}

En la tabla 8, se muestran los valores de las medidas de desempeño propuestas para cada una de las 18 configuraciones encontradas. Estos datos son insumo para el análisis jerárquico de decisiones.

TABLA VIII.

VALORES DE LAS MEDIDAS DE DESEMPEÑO PROPUESTAS

\begin{tabular}{|c|c|c|c|c|c|c|c|c|}
\hline $\mathbf{P}$ & $\begin{array}{c}\text { Confi } \\
\text { gurac } \\
\text { ión }\end{array}$ & $\mathbf{U}$ & $\mathbf{S}$ & M & $\mathbf{T}$ & $\begin{array}{l}\text { IM } \\
\text { (und) }\end{array}$ & DK & $\underset{(\min )}{\mathbf{C M}}$ \\
\hline \multirow{5}{*}{1} & \multirow{2}{*}{1} & 59,5 & 100 & \multirow{3}{*}{0,38} & 20,0 & \multirow{3}{*}{170} & 53,8 & \multirow{3}{*}{7500} \\
\hline & & $1 \%$ & $\%$ & & $7 \%$ & & $5 \%$ & \\
\hline & \multirow{2}{*}{1} & 55,7 & 100 & & 18,8 & & 53,8 & \\
\hline & & $9 \%$ & $\%$ & \multirow{2}{*}{0,32} & $1 \%$ & 170 & $5 \%$ & \multirow[t]{2}{*}{8000} \\
\hline & \multirow{2}{*}{2} & 59,5 & 100 & & 20,0 & & 53,8 & \\
\hline \multirow{5}{*}{2} & & $1 \%$ & $\%$ & \multirow[t]{2}{*}{0,38} & $7 \%$ & 170 & $5 \%$ & \multirow[t]{2}{*}{7500} \\
\hline & \multirow{2}{*}{3} & 55,7 & 100 & & 18,8 & & 51,2 & \\
\hline & & $9 \%$ & $\%$ & \multirow[t]{2}{*}{0,22} & $1 \%$ & 170 & $8 \%$ & \multirow[t]{2}{*}{8000} \\
\hline & \multirow{2}{*}{4} & 55,7 & 100 & & 18,8 & & 51,2 & \\
\hline & & $9 \%$ & $\%$ & 0,22 & $1 \%$ & 170 & $8 \%$ & \multirow[t]{2}{*}{8000} \\
\hline \multirow{8}{*}{3} & \multirow{2}{*}{1} & 55,7 & 100 & & 18,8 & & 41,0 & \\
\hline & & $9 \%$ & $\%$ & 0,38 & $1 \%$ & 170 & $3 \%$ & \multirow[t]{2}{*}{8000} \\
\hline & \multirow{2}{*}{2} & 55,7 & 100 & & 18,8 & & 51,2 & \\
\hline & & $9 \%$ & $\%$ & 0,32 & $1 \%$ & 170 & $8 \%$ & \multirow[t]{2}{*}{8000} \\
\hline & \multirow{2}{*}{3} & 52,5 & 100 & & 17,7 & & 43,5 & \\
\hline & & $1 \%$ & $\%$ & 0,39 & $1 \%$ & 170 & $9 \%$ & \multirow[t]{2}{*}{8500} \\
\hline & \multirow{2}{*}{4} & 52,5 & 100 & & 17,7 & & 35,9 & \\
\hline & & $1 \%$ & $\%$ & 0,22 & $1 \%$ & 170 & $0 \%$ & \multirow[t]{2}{*}{8500} \\
\hline \multirow{6}{*}{4} & \multirow{2}{*}{5} & 55,7 & 100 & & 18,8 & & 56,4 & \\
\hline & & $9 \%$ & $\%$ & 0,55 & $1 \%$ & 170 & $1 \%$ & \multirow{2}{*}{8000} \\
\hline & \multirow{2}{*}{1} & 59,5 & 100 & & 20,0 & & 61,5 & \\
\hline & & $1 \%$ & $\%$ & 0,32 & $7 \%$ & 170 & $4 \%$ & \multirow[t]{2}{*}{7500} \\
\hline & \multirow[b]{2}{*}{2} & 59,5 & 100 & & 20,0 & & 58,9 & \\
\hline & & $1 \%$ & $\%$ & 0,16 & $7 \%$ & 170 & $7 \%$ & 7500 \\
\hline
\end{tabular}

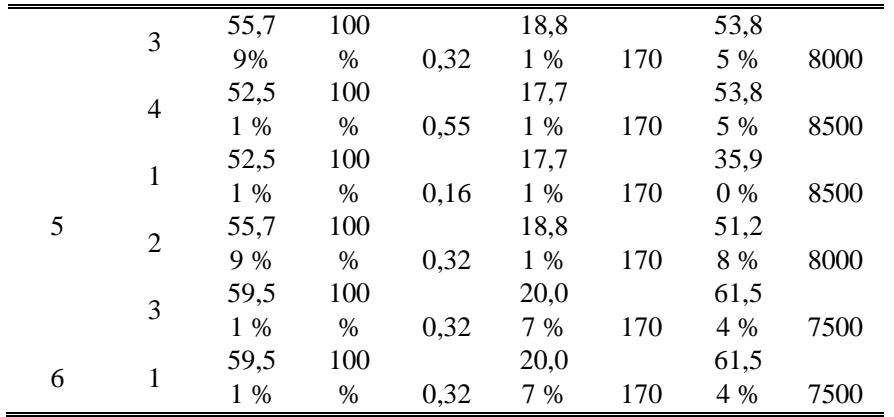

\section{E. Proceso de análisis jerárquico}

En la tabla 9, de acuerdo al comportamiento presentado por las medidas de desempeño propuestas, se halló el vector prioridad. Es de notar que el criterio con más peso en este vector es la Utilización Global y el que presenta menor peso porcentual es el Inventario máximo.

\begin{tabular}{cc} 
TABLA IX & \\
VECTOR PRIORIDAD & \\
\hline \hline $\begin{array}{c}\text { Medida de } \\
\text { desempeño } \\
\text { Utilización Global }\end{array}$ & Valor \\
Nivel de Servicio & $38 \%$ \\
Movimiento Entre Células & $15 \%$ \\
Tiempo en Inventario & $8 \%$ \\
Inventario Máximo & $9 \%$ \\
Desviación entre Grupos & $7 \%$ \\
Capacidad de las Máquinas en los Grupos & $11 \%$ \\
\hline \hline
\end{tabular}

En la Tabla 10 es presentado el puntaje final de las opciones de configuración disponible, obtenido como la suma ponderada, por el vector prioridad, de los valores normalizados de las medidas de desempeño para cada una de las opciones.

TABLA X PUNTAJE FINAL DE LAS OPCIONES DE CONFIGURACIÓN

$\begin{array}{ccc}\text { Valores de P } & \text { Configuración } & \text { Puntaje } \\ 1 & 1 & 61,41 \% \\ & 1 & 60,10 \% \\ 2 & 2 & 61,44 \% \\ & 3 & 59,07 \% \\ 3 & 4 & 59,07 \% \\ & 1 & 55,30 \% \\ & \mathbf{2} & \mathbf{6 8 , 9 2} \% \\ & 3 & 54,73 \% \\ & 4 & 52,59 \% \\ & 5 & 62,21 \% \\ \end{array}$




\begin{tabular}{lll}
\hline \hline & 2 & $60,20 \%$ \\
& 3 & $60,11 \%$ \\
& 4 & $57,11 \%$ \\
5 & 1 & $52,09 \%$ \\
& 2 & $59,87 \%$ \\
& 3 & $61,79 \%$ \\
6 & 1 & $61,79 \%$ \\
\hline \hline
\end{tabular}

En AHP se dice que el decisor, o persona que introduzca los juicios, es consistente si la matriz de comparaciones pareadas lo es. Esto se verifica a través de la razón de consistencia (RC), la cual es el cociente entre el índice de consistencia de la instancia (IC) y el índice consistencia aleatorio (IA) [22].

Recordemos que el índice de consistencia aleatorio (IA) se define como el índice de consistencia aleatorio medio obtenido mediante la simulación de 100.000 matrices recíprocas generadas aleatoriamente utilizando la escala de Saaty mientras entre el índice de consistencia de la instancia (IC) es el valor obtenido a partir de los datos del caso en estudio.

La razón de consistencia de esta investigación fue de 0.06 , menor al parámetro 0.10 que determina la metodología AHP. Por ello se puede concluir que los juicios con los cuales se realizaron las comparaciones pareadas de los criterios de evaluación (medidas de desempeño propuesta) son consistentes y pueden los resultados obtenidos considerarse validos con un alto grado de confianza.

\section{DISCUSIÓN}

\section{A. Análisis de resultados del momento propuesto}

A continuación, se presentarán los principales resultados y hallazgos donde se validará la metodología propuesta en este documento.

El comportamiento de la solución óptima, valorada en costo (unidades monetarias), del modelo propuesto muestra un comportamiento creciente de la solución a medida que aumenta el número de familias a crear (p) con cada una de las opciones, puede ser observado en la figura 7.

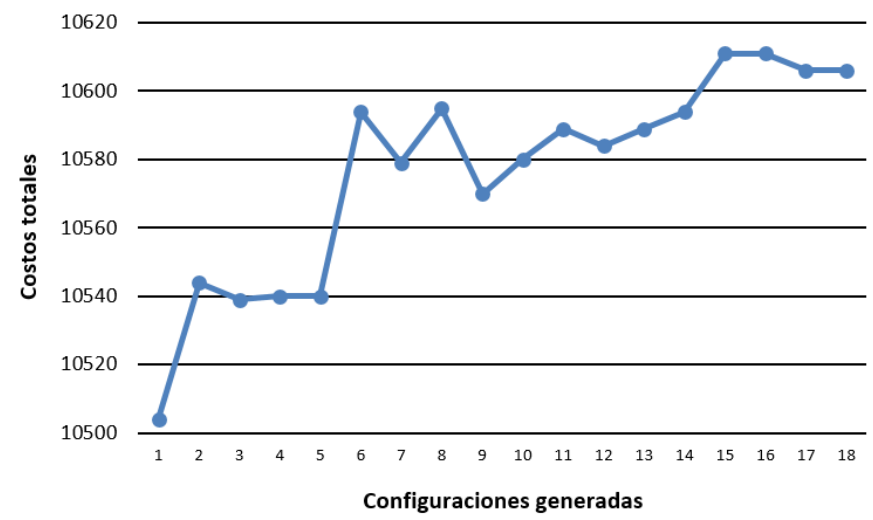

Fig. 7. Solución óptima del modelo propuesto: Costo total vs Configuración.

La función objetivo del modelo propuesto se compone de varios factores de costos que influyen sobre este comportamiento, y uno de ellos que se encuentra directamente relacionado con el número de familias. La diferencia básica entre las soluciones óptimas la establece la componente de costo de alistamiento y costos de transporte de material entre los grupos puesto que la asignación obtenida implica movimiento de operaciones de un mismo producto entre grupos para todas las opciones generadas.

De ser considerado el costo como medida de desempeño, la configuración a seleccionar estaría conformado por una única familia de productos. La tecnología de grupos no estaría reflejada y las ventajas que podrían adquirirse por trabajar en talleres más especializados se perdería. Es de notar que el costo de la flexibilidad implica un incremento monetario de hasta el $1.14 \%$ (cambio en el costo total entre la configuración con menor costo a la de mayor costo) incremento de magnitud baja si se reflexiona sobre las características (reflejadas en los criterios de desempeño propuestos) que pueden adquirir los talleres de trabajos si adaptan su enfoque.

\section{B. Plan definitivo de configuración de planta, capacidad y producción}

La mejor configuración posible de célula de manufactura a partir de las medidas de desempeño propuestas y la metodología AHP es la configuración 2 cuando $\mathrm{P}$ toma el valor de 3 (ver Figura 8) y donde la distribución de los productos en las familias queda establecida de la siguiente forma: $F 1=\{P 1 P 3$ $\mathrm{P} 4\}, \mathrm{F} 2=\{\mathrm{P} 2 \mathrm{P} 6\}$ y F3 $=\{\mathrm{P} 5\}$. 
FAMILIA 1

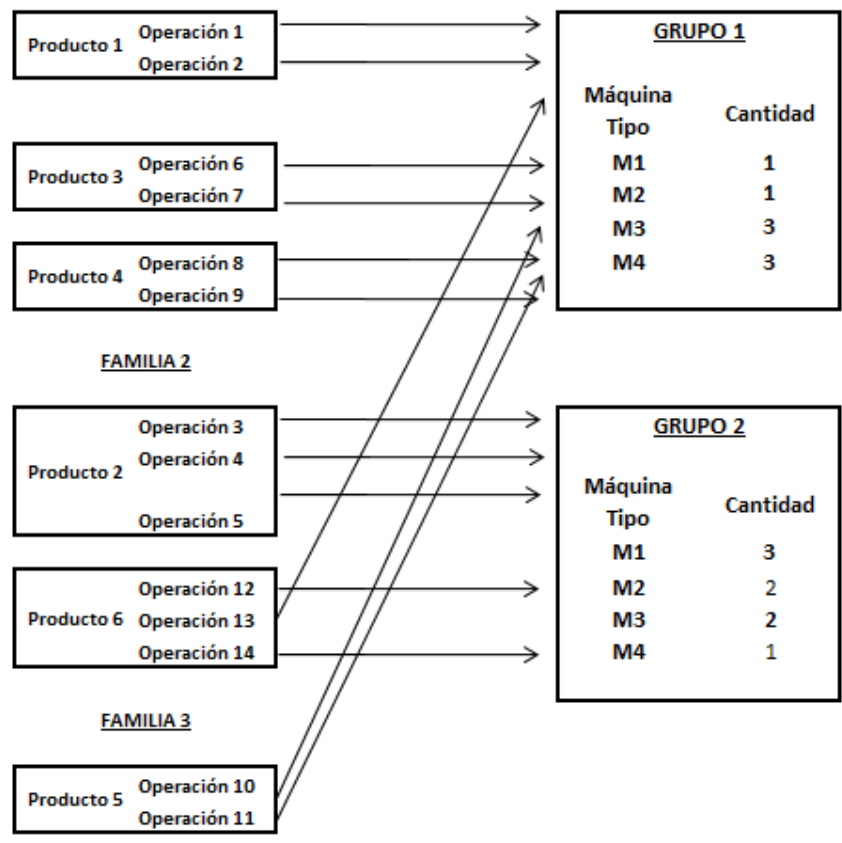

Fig. 8. Configuración seleccionada a partir de la metodología AHP. Fuente: Elaboración propia.

Los coeficientes que generaron esta opción fueron los Coeficientes de Hamann, de Yule, y de Simple Matching. En la tabla 11 puede observarse la designación de operaciones a cada uno de los grupos formados dentro de la configuración.

TABLA XI

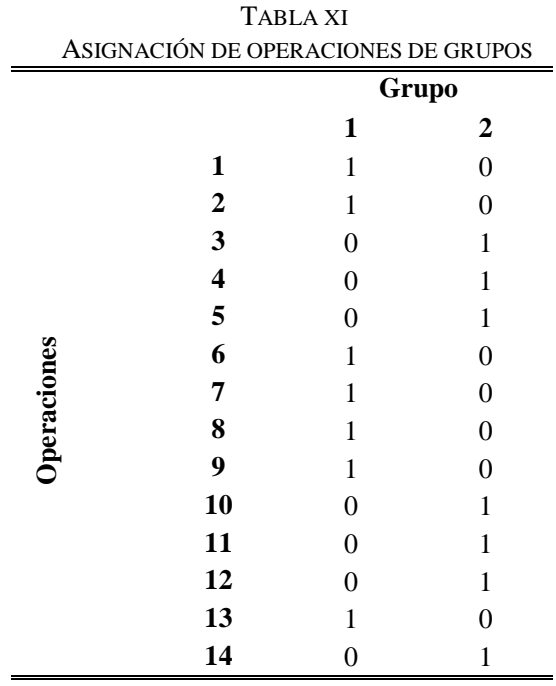

En la tabla 12 se encuentran el número de máquinas de cada tipo que fueron asignadas a cada grupo. No fueron generadas unidades pendientes a entregar ni inventario entre periodos del horizonte de planeación con la configuración seleccionada, el plan de producción por tipo de producto y periodo se detalla en la tabla 13.
TABLA XII

NÚMERO DE MÁQUINAS TIPO ASIGNADAS POR GRUPO

\begin{tabular}{|c|c|c|c|}
\hline \multirow{6}{*}{ 莺 } & & \multicolumn{2}{|c|}{ Grupo } \\
\hline & & 1 & 2 \\
\hline & 1 & 1 & 3 \\
\hline & 2 & 1 & 2 \\
\hline & 3 & 3 & 2 \\
\hline & 4 & 3 & 1 \\
\hline
\end{tabular}

TABLA XIII

UNIDADES PRODUCIDAS POR PRODUCTO Y PERIODO

\begin{tabular}{|c|c|c|c|c|c|c|c|}
\hline & \multicolumn{6}{|c|}{ Periodos } \\
\hline & & 1 & 2 & 3 & 4 & 5 & 6 \\
\hline \multirow{6}{*}{$\begin{array}{l}\frac{\tilde{0}}{0} \\
\frac{0}{0} \\
0\end{array}$} & Revistas & 125 & 45 & 100 & 130 & 45 & 100 \\
\hline & Volantes & 140 & 50 & 100 & 150 & 50 & 100 \\
\hline & Plegables & 205 & 50 & 200 & 210 & 50 & 200 \\
\hline & Carpetas & 145 & 65 & 120 & 150 & 65 & 120 \\
\hline & Libros & 165 & 70 & 100 & 120 & 40 & 150 \\
\hline & Cuadernos & 195 & 50 & 150 & 110 & 50 & 100 \\
\hline
\end{tabular}

\section{Análisis de sensibilidad del AHP}

El objetivo del análisis es probar la robustez del modelo ante cambios en los parámetros del AHP. Para esto, se realiza variando la matriz de preferencia de los criterios, puesto que esta se hace de manera subjetiva a juicio del analista la idea es mirar el comportamiento cuando esta se cambia.

Se realizaron tres variaciones (cada una de forma individual) en la matriz de criterios: Nivel de servicio, Capacidad de las máquinas e Inventario Máximo. Donde se otorgó mayor importancia a cada uno de estos criterios a través de variaciones en la magnitud de cada criterio de la matriz de preferencias.

A raíz de lo anterior, en la tabla 14 podemos observar los cambios generados en los vectores prioridad. En general, para los tres casos, se observa una disminución en la importancia del criterio Utilización global el cual pasó de $38 \%$ a alrededor de $20 \%$.

TABLA XIV

CAMBIOS EN VECTORES PRIORIDAD

\begin{tabular}{cccc}
\hline \hline $\begin{array}{c}\text { Medida de } \\
\text { desempeño }\end{array}$ & $\begin{array}{c}\text { Nivel de } \\
\text { Servicio }\end{array}$ & $\begin{array}{c}\text { Capacidad de } \\
\text { las Máquinas } \\
\text { en los Grupos }\end{array}$ & $\begin{array}{c}\text { Inventario } \\
\text { Máximo }\end{array}$ \\
$\begin{array}{c}\text { Utilización } \\
\text { Global }\end{array}$ & $22 \%$ & $19 \%$ & $19 \%$ \\
$\begin{array}{c}\text { Nivel de } \\
\text { Servicio } \\
\text { Movimiento }\end{array}$ & $\mathbf{2 5 \%}$ & $20 \%$ & $16 \%$ \\
Entre Células & $9 \%$ & $9 \%$ & $6 \%$ \\
\hline \hline
\end{tabular}




\begin{tabular}{cccc}
\hline \hline Tiempo en & $10 \%$ & $10 \%$ & $9 \%$ \\
$\begin{array}{c}\text { Inventario } \\
\text { Inventario } \\
\text { Máximo }\end{array}$ & $8 \%$ & $8 \%$ & $\mathbf{2 1 \%}$ \\
$\begin{array}{c}\text { Desviación entre } \\
\text { Grupos }\end{array}$ & $12 \%$ & $12 \%$ & $14 \%$ \\
$\begin{array}{c}\text { Capacidad de las } \\
\text { Máquinas en los } \\
\text { Grupos }\end{array}$ & $14 \%$ & $\mathbf{2 2 \%}$ & $14 \%$ \\
\hline \hline
\end{tabular}

En los tres casos, a pesar del cambio en las preferencias en la matriz de los criterios el AHP la mejor configuración que se puede realizar continúa siendo la configuración 2 cuando $\mathrm{P}$ toma el valor de 3 seguida la configuración 5 en el mismo valor de $\mathrm{P}$, a dos puntos porcentuales de diferencia. Una brecha menor al de la instancia original, la cual se ubicaba a 6 puntos porcentuales. Es de resaltar que el índice de consistencia obtenido siempre fue menor a 0.1. Por lo tanto, podemos concluir que las variaciones individuales en los criterios de las configuraciones candidatas no modifican la decisión encontrada la configuración seleccionada es dominante en relación a las demás opciones y la metodología presentada es consistente.

\section{CONCLUSIONES}

El desarrollo de esta metodología permitió combinar la optimización con la técnica de tecnología de grupo introduciendo múltiples coeficientes de similaridad y evaluando medidas de desempeño propuestas mediante la metodología AHP de forma consistente. Lo cual, en otros casos, son valoradas según la percepción de uno o varios expertos implicando posible sesgo en la valoración de decisiones con componente de carácter cuantitativas (no fácilmente comparables entre ellas) y cualitativos.

Lo anterior es la principal fortaleza de la metodología propuesta, ya que los indicadores con criterios medibles a partir de resultados del problema. Por lo tanto, se reducen las inconsistencias naturales del juicio de expertos y por ello los índices de consistencia bajos.

El comportamiento de la solución óptima del modelo propuesto muestra un comportamiento creciente de la solución a medida que aumenta el número de familias a crear (p) con cada una de las opciones. La diferencia básica entre las soluciones óptimas la establece la componente de costo de alistamiento y costos de transporte de material entre los grupos puesto que la asignación obtenida implica movimiento de operaciones de un mismo producto entre grupos para todas las opciones generadas.

Es posible abordar el problema de la formación de células considerando un enfoque en el que se descompone el problema en sub problemas menos complejos. Y así poder incluir al tiempo múltiples criterios de agrupación orientados al taller, la integración con el plan de producción y plan de capacidad a mediano plazo logrando propuestas de configuración adecuadas al entorno donde sea aplicado.

Los problemas que logren aproximarse a la optimalidad son interesantes desde el punto de vista académico, pero su utilidad práctica y adaptabilidad a situaciones reales llegar a ser muy limitada. De esta manera puede comprenderse la dimensión real del problema de planeación de configuración, capacidad y producción de planta y por consiguiente la necesidad de estudiar métodos que contemplen nuevas propuestas en cuanto a su resolución.

\section{REFERENCIAS}

[1] J. Bravo, J. P. Orejuela, and Z. Menduiña, "Aproximación a la medición del impacto del Backorder en sistemas de manufactura flexible," Rev. Fac. Ing. Univ. Antioquia, no. 65, pp. 99-111, 2012.

[2] T. Aljuneidi and A. A. Bulgak, "A mathematical model for designing reconfigurable cellular hybrid manufacturingremanufacturing systems," Int. J. Adv. Manuf. Technol., pp. 1-12, 2016. DOI: $10.1007 / \mathrm{s} 00170-016-9141-\mathrm{z}$.

[3] N. Aghajani-Delavar, E. Mehdizadeh, S. A. Torabi, and R. C Tavakkoli-Moghaddam, "Design of a new mathematical model for integrated dynamic cellular manufacturing systems and production planning,” Int. J. Eng. Trans. B Appl., vol. 28, no. 5, pp. 746-754, 2015. DOI: 10.5829/idosi.ije.2015.28.05b.13

[4] K. Deep and P. K. Singh, "Design of robust cellular manufacturing system for dynamic part population considering multiple processing routes using genetic algorithm," J. Manuf. Syst., vol. 35, pp. 155163, 2015. DOI: 10.1016/j.jmsy.2014.09.008.

[5] V. R. Ghezavati, "Designing integrated cellular manufacturing systems with tactical decisions," J. Chinese Inst. Eng. Trans. Chinese Inst. Eng. A/Chung-kuo K. Ch'eng Hsuch K'an, vol. 38, no. 3, pp. 332-341, 2015. DOI: 10.1080/02533839.2014.970350.

[6] A. Hassan Zadeh, H. Afshari, and R. Ramazani Khorshid-Doust, "Integration of process planning and production planning and control in cellular manufacturing," Prod. Plan. Control, vol. 25, no. 10, pp. 840-857, 2013. DOI: 10.1080/09537287.2013.767394.

[7] A. K. Chakravarty and A. Shtub, "Production Scheduling During Phased Implementation of Flexible Manufacturing Cells," IIE Trans., vol. 22, no. 4, pp. 292-299. DOI: $10.1080 / 07408179008964184$.

[8] E. Pan, G. Wang, L. Xi, L. Chen, and X. Han, "Single-machine group scheduling problem considering learning, forgetting effects and preventive maintenance," Int. J. Prod. Res., vol. 52, no. 19, pp. 5690-5704, 2014. DOI: 10.1080/00207543.2014.904967.

[9] Y. Gholipour-Kanani, N. Aghajani, R. Tavakkoli-Moghaddam, and S. Sadinejad, "Proposed a novel group scheduling problem in a cellular manufacturing system," in 19th International Conference on Industrial Engineering and Engineering Management: Assistive Technology of Industrial Engineering, 2013, pp. 843-853. DOI: 10.1007/978-3-642-38391-5-89.

[10] J. Deuse, B. Konrad, and F. Bohnen, "Renaissance of group technology: Reducing variability to match lean production prerequisites," in IFAC Proceedings Volumes (IFAC- 
PapersOnline), 2013, pp. 998-1003. DOI: 10.3182/20130619-3RU-3018.00319.

[11] P. E. Ibrahim, Y., Alqattan, "Systematic approach to cellular manufacturing systems design," J. Mech. Work. Technol., vol. 2, pp. 415-424, 1989.

[12] S. Carmo-Silva, M. C. Morais, and F. A. Fernandes, "A practical methodology for cellular manufacturing systems design - An industrial study," Rom. Rev. Precis. Mech. Opt. Mechatronics, no. 41, pp. 103-118, 2012. https://www.scopus.com/inward/record.uri?eid=2-s2.084865589708\&partnerID $=40 \& \mathrm{md} 5=\mathrm{fa} 010 \mathrm{bb} 4 \mathrm{c} 327292 \mathrm{ba} 2 \mathrm{e} 16 \mathrm{~b} 549$ be2dd69.

[13] R. Kia, A. Baboli, N. Javadian, R. Tavakkoli-Moghaddam, M. Kazemi, and J. Khorrami, "Solving a group layout design model of a dynamic cellular manufacturing system with alternative process routings, lot splitting and flexible reconfiguration by simulated annealing," Comput. Oper. Res., vol. 39, no. 11, pp. 2642-2658, 2012. DOI: 10.1016/j.cor.2012.01.012.

[14] R. Manzini, F. Bindi, and A. Pareschi, The threshold value of group similarity in the formation of cellular manufacturing systems, vol. 48, no. 10. 2010. DOI: 10.1080/00207540802644860.

[15] M. Cantamessa and A. Turroni, "A pragmatic approach to machine and part grouping in cellular manufacturing system design," Int. J. Prod. Res., vol. 35, no. 4, pp. 1031-1050, 1997. DOI: $10.1080 / 002075497195524$.

[16] R. Kia et al., "A multi-objective mathematical model for cellular manufacturing systems design with probabilistic demand and machine reliability analysis,” Appl. Mech. Mater., vol. 685, no. 1, pp. 103-118, 2014. DOI: 10.1504/IJSOM.2015.067479.

[17] S. Wei and H. Zhao, "Research of the optimal production planning based on multi-group," Appl. Mech. Mater., vol. 263-266, no. PART 1, pp. 3490-3495, 2013. DOI: 10.4028/www.scientific.net/AMM.263-266.3490.

[18] F. T. S. Chan and K. Abhary, "Design and evaluation of automated cellular manufacturing systems with simulation modelling and AHP approach: A case study," Integr. Manuf. Syst., vol. 7, no. 6, pp. 3952, 1996. DOI: 10.1108/09576069610151176.

[19] [D. Riaño, Análisis de conglomerados, 1st ed. Bogota, 2001.

[20] Y. Yin and K. Yasuda, "Similarity coefficient methods applied to the cell formation problem: A taxonomy and review," Int. J. Prod. Econ., vol. 101, no. 2, pp. 329-352, 2006.

[21] A. Kusiak, Computational Intelligence in Design and Manufacturing. New York: John Wiley \& Sons, Inc., 2000. DOI: 10.1016/j.ijpe.2005.01.014.

[22] R. W. Saaty, "The analytic hierarchy process-what it is and how it is used," Math. Model., vol. 9, no. 3-5, pp. 161-176, 1987. DOI: 10.1016/0270-0255(87)90473-8.

A. M. Castillo-Zapata was born in Cali, Valle del Cauca, Colombia. She received the degree in industrial engineering from the Universidad del Valle, Colombia, in 2010. Since his degree he has worked in different companies in operations planning and control. His interest areas are, the planning of operations, logistics and transport.

J. P. Orejuela-Cabrera is Full professor of the school of Industrial Engineering at Universidad del Valle (Cali, Colombia). He has a Master degree in industrial engineering from and Industrial Engineer, from the Universidad del Valle. His current research interests: Applied mathematical modeling. Supply chain management and Simulation of production and logistics systems. He is member of the Research Group: Logistica y producción from the Universidad del Valle.

C. Andrés Gil-González is Phd student from Universidad de Chile, in the Faculty of Physical Sciences and Mathematics. He has a Master degree in industrial engineering from the Universidad del Valle, Cali, Colombia. His research interests include Operations Research, Scheduling, Combinatorial Optimization, Metaheuristics, Supply Chain Management, Logistics and Operations Management. 